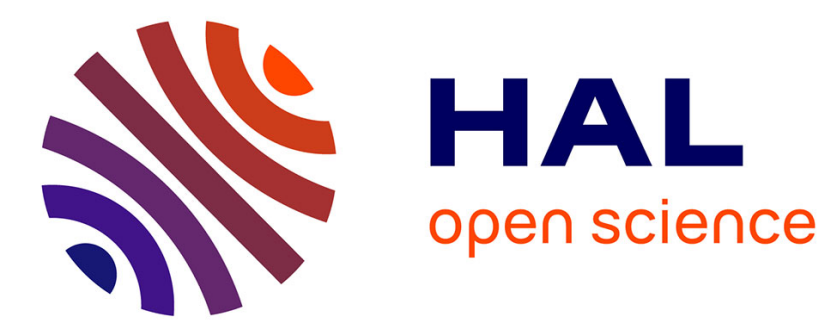

\title{
Binding metals to polymers. A short review of basic physical mechanisms
}

\author{
B. Escaig
}

\section{To cite this version:}

B. Escaig. Binding metals to polymers. A short review of basic physical mechanisms. Journal de Physique IV Proceedings, 1993, 03 (C7), pp.C7-753-C7-761. 10.1051/jp4:19937120 • jpa-00251739

\section{HAL Id: jpa-00251739 https://hal.science/jpa-00251739}

Submitted on 1 Jan 1993

HAL is a multi-disciplinary open access archive for the deposit and dissemination of scientific research documents, whether they are published or not. The documents may come from teaching and research institutions in France or abroad, or from public or private research centers.
L'archive ouverte pluridisciplinaire HAL, est destinée au dépôt et à la diffusion de documents scientifiques de niveau recherche, publiés ou non, émanant des établissements d'enseignement et de recherche français ou étrangers, des laboratoires publics ou privés. 


\title{
Binding metals to polymers. A short review of basic physical mechanisms
}

\author{
B. ESCAIG
}

Laboratoire de Structures et Propriétés de l'Etat Solide, URA 234 du CNRS, Université des Sciences et Technologies de Lille, 59655 Villeneuve d'Ascq cedex, France

\begin{abstract}
Normally metals do not bond to polymers. Binding metals to polymers is a complex phenomena which brings into play interfering modifications at different places : at the adhesive interface and within a thicker interphase layer. Bond formation at the interface is obtained by functionalisation of the polymer surface to make acid-base reactions with the metallic oxide (mainly hydrogen bonding with alumina). However, common practice shows that the main resistance to delamination stems from dissipation processes in the interphase region, the importance of which is tremendous for a strong adherence. Dissipation is conveniently modelled by the fracture mechanics parameter $G$, the energy release rate, and the way it can be built up from recent basic physical mechanisms is reviewed, either as a plastic dissipation (suction of polymer chains at crack tip) or as viscoelastic losses (far from the tip). From these models, some experimental features begin to be understood.
\end{abstract}

\section{Introduction}

Binding metals to polymers is for long a process widely used in industrial applications. However the trends needed for understanding the basic physical mechanisms begin to be worked out only recently, eventhough most of the chemical features of surface interactions have been elucidated since the two last decades. This review is mainly aimed at a survey of the present physics of these phenomena, focussing on the concepts which are the most useful for guiding practice, that is to say, for the global behaviour of the adhesive junction. The thermodynamical approach is useful first to clear out the respective role either of chemical bonds ( $w$, the DUPRE reversible work of adhesion) or of energy dissipations (G, the release rate of energy) in the adherence force between a polymer and a metal. $G$ and $w$ are related to different parts in the material : while $w$ addresses only to the interface area, $G$ addresses to a rather thick "interphase" layer (about 0.1 or $1 \mathrm{~mm}$ thick). The next two paragraphs deal with these two regions : the bond formation at the interface, and the energy dissipation in the interphase region. Finally, a few theoretical remarks are given in the last part, on the mechanical performance of the adhesive junction. 


\section{Thermodynamics}

It is well known that the amount of energy contributed by surface Gibbs enthalpies for separating two solids, noted 1 and 2, is the DUPRE reversible work $w$ :

$$
\mathrm{w}=\gamma_{1}+\gamma_{2}-\gamma_{12}
$$

with $\gamma_{1}, \gamma_{2}$ the surface tensions and $\gamma_{12}$ the interfacial energy of the boundary between (1) and (2). This change in energy of chemical bonds is localised very near the surface, within a few atomic spacings in the simplest quasi-chemical model.

However, the external work needed in reality to separate two solids is much larger than $\mathrm{w}$ by two or three orders of magnitude. This paradox has been solved by the fracture mechanics approach [1] which defines $G$, the fracture energy or the strain energy release rate, as the most important quantity.

Let $\mathcal{G}(\mathrm{T}, \mathrm{P}, \mathrm{A})$ be the Gibbs enthalpy of the system of two solids at temperature $T$, under the load $P$ ( $u$ being the load displacement), with $A$ the adhesive junction area, $G(T, P, A)=$ U-TS-Pu. Simple thermodynamics shows that, for an elementary change in $T, P, A$ :

with

$$
\mathrm{d} g=-\mathrm{SdT}-\mathrm{udP}+(\mathrm{G}-\mathrm{w}) \mathrm{dA}
$$

where the first term in $G$ is the increase in elastic energy of solids, and the second is the increase (negative, in general) in the potential energy of the external mechanical setting.

For the case $G>\mathrm{w}$ under $T, P$ constant, the crack extends $(\mathrm{d} A<0)$, while $\mathrm{G}=\mathrm{w}$ is the GRIFFITH criterion for crack equilibrium. Now assume that for the special load value $P=P_{c}$ one has :

$$
\mathrm{G}\left(\mathrm{P}_{\mathrm{c}}\right)=\mathrm{w} \text { and }(\partial \mathrm{G} / \partial \mathrm{A})_{\mathrm{T}, \mathrm{P}_{\mathrm{C}}}<0
$$

i.e. that $G$ increases when the crack starts extending, or the crack equilibrium is unstable, then $P_{c}$ is called the elastic adherence force.

Finally, $G$ is related to the stress intensity factor $K$, just as in usual fracture mechanics. For exemple in mode I :

$$
\begin{aligned}
& \mathrm{G}=0.5 \mathrm{~K}_{1}^{2}\left[\left(1 / \mathrm{E}_{1}\right)+\left(1 / \mathrm{E}_{2}\right)\right] \\
& \mathrm{G}=0.5 \mathrm{~K}_{1}^{2}\left[\left(1-v_{1}^{2} / \mathrm{E}_{1}\right)+\left(1-v_{2}^{2} / \mathrm{E}_{2}\right)\right]
\end{aligned}
$$

in plane stress conditions

in plane strain conditions with $\mathrm{E}$ the Young

modulus and $v$ the Poisson modulus.

It is worthy to note that, unlike $w$ which addresses to the chemistry of the interface area, $G$ relates to the physical phenomena in a rather tick "interphase" layer (up to 0.1 or $1 \mathrm{~mm}$ ). These two topics are now reviewed.

\section{Bond formation at the Interface}

It is clear that adhesion needs molecular bonding. Since molecular forces are relatively short range, close contact and good wetting conditions are required. In the case of polymers and metals, i.e. metallic oxides, this is hardly the case : polymers have a poor wettability from their low surface tensions $\gamma$ (polyolefins and polysiloxanes are worst, polyesters and epoxys are a little better, see [2a] for a review), while metallic oxide surfaces may often exhibit a pitfall topology making the intimate contact of molecules difficult. Common practice improves the situation : polymeric primaries with low viscosities, thermal stimulation of molecular mobilities (i.e. thermoplastic adhesives), polymer surface excitation to increase functional reactive groups (hydroxil, hydroperoxide, carboxylic species) by flaming, Corona discharge or even plasma treatment, and finally, chemical functionalisation of polymer surfaces by grafting. An example of the latter technique is given in [3], where a polypropylene film is grafted with acrylic acid after an oxidising 
electron beam irradiation, resulting into a surface copolymer with polyacrylic acid $(\mathrm{n}=2,3,4, \ldots)$ :

$$
\begin{gathered}
-\mathrm{CH}_{2}-\mathrm{CH}\left(\mathrm{CH}_{3}\right)-\mathrm{CH}_{2}-\mathrm{C}\left(\mathrm{CH}_{3}\right)-\mathrm{CH}_{2}-\mathrm{CH}\left(\mathrm{CH}_{3}\right)- \\
\mathrm{O} f \mathrm{CH}_{2}-\mathrm{CH}+\frac{1}{1} \\
\text { COOH }
\end{gathered}
$$

Chemical bonding can be generated through different forces :

(1) Van der Waals forces originate from molecular dipole interactions. When integrated over the two approaching surfaces, they turn out to be a weak but long range interaction (decreasing as $\mathrm{r}^{-2}$ or $\mathrm{r}^{-3}$ beyond $10 \mathrm{~nm}$, see [2b]).

(2) Acid-Base phenomena [2c] encompass all charge transfer reactions : hydrogen bonding, and the electron pair donor-acceptor interactions involving both covalent and ionic factors (or equivalently, electron-hole pair formation in the energy bands - conduction and valence bands - of the two insulating solids, see [4]). In the case of the above grafted PP films on aluminium, ROMERO has shown in [3] that hydrogen bonds formed between the (polymer) carboxylic and the (oxide) hydroxil groups are prevalent : an infrared absorption at about $\lambda=1740 \mathrm{~cm}^{-1}$ is characteristic of the interface formation; much less important is a secondary binding, also observed, through a covalent coordination bond between alumina $\left(\mathrm{Al}^{3+}\right)$ and the carboxylate groups (COO-) present at the polymer surface $\left(\lambda=1600 \mathrm{~cm}^{-1}\right)$.

\section{Energy dissipation in the interface region}

Dissipation is by far the main resistance to delamination. Adhesives are designed to yield at energies much higher than just the bond interfacial energy. Breaking the adhesive junction is basically the same phenomenon as breaking one solid in bulk : in both cases two main sources of dissipation are distinguished, the plastic and the viscoelastic energy losses, taking place in quite different regions. In a similar way, both fracture energies $G$ depend on crack

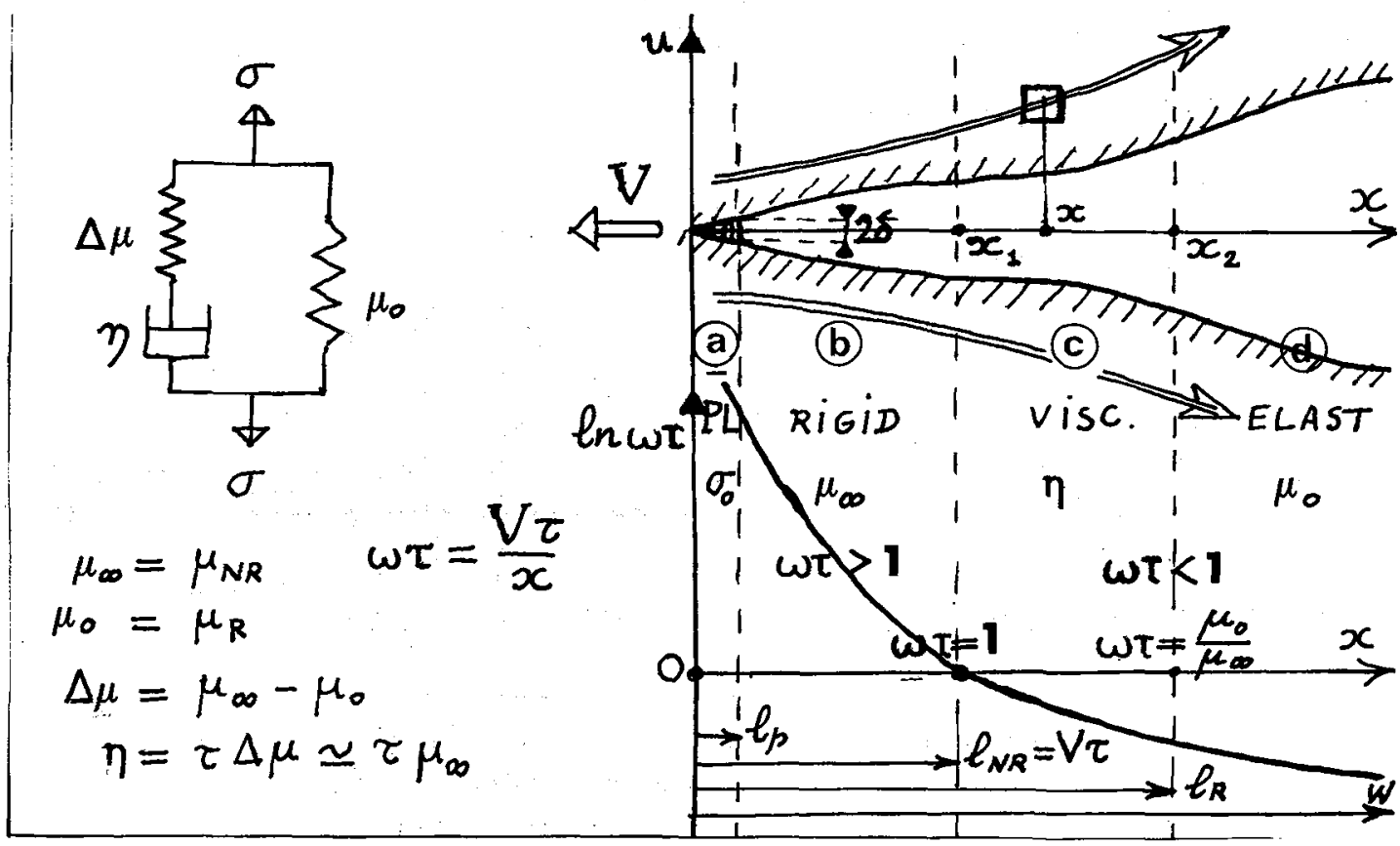

Fig. 1 : Rheological model of a propagating crack at rate V [5] 
rate $V$ at high values, $G=G_{0} f(V)$ with $f(0)=1$. We discuss below each of the two loss mechanisms, but we need first to know at what places they occur.

\subsection{Different energy losses at different places along the crack}

Figure 1 shows a crack running at rate $V$ through an elasto-viscoplastic solid of yield stress $\sigma_{0}$, the viscoelastic behaviour of which is modelled simply by the standard linear solid with three parameters : $\mu_{0}$, the relaxed modulus, $\mu_{\infty}$ the non-relaxed modulus, and the viscosity $\eta$. With the polymer as solid, this might depict the case of a cohesive fracture ; alternatively, the rheology of an advancing crack along an adhesive boundary between a polymer and a metal (adhesive fracture) would not be basically different from this picture, as noted above, using simply specific parameters for each of the two crack halves.

It is well known that in such a solid, elastic stresses start relaxing beyond a time $\tau=\Delta \mu / \eta$ (fig. 1) while the elastic strain lags behind the stress until a time $\tau_{\mathrm{d}}=\tau\left(\mu_{\infty} / \mu_{0}\right)$, or delay time, is elapsed, beyond which the phase lag vanishes. Thus, strain energy is dissipated only in the time interval $\tau_{d}-\tau$. Let us assume now, instead of a moving crack, a crack at rest in a flowing medium. An elementary volume of matter at $x$ would have been sustaining the crack stress field for a time $x / V$. Then, viscoelastic dissipation in this small volume occurs between boundaries :

$$
x_{1}=\ell_{N R}=V_{\tau} \text {, and } x_{2}=\ell_{R}=V \tau_{d}=V_{\tau}\left(\mu_{\infty} / \mu_{0}\right)
$$

(see in fig. 1), that is to say in a region remote from the crack tip, as DE GENNES has first pointed out [5]. Finally, there exists at the crack tip a plastically strained region over a length $\ell_{p}$, given in the IRWIN model by $\ell_{p} \cong \mu_{\infty}\left(2 \delta / \sigma_{0}\right)$ with $2 \delta$, the crack opening displacement [6], here, the length of extended chains at the tip. In conclusion, we end up with two dissipative regions along the crack : (i) plastic dissipation at the crack tip over the length $\ell_{p}$ (region (a) in fig. 1); and (ii) viscoelastic dissipation at longer range, at distances between $V \tau$ and $V \tau\left(\mu_{\infty} / \mu_{0}\right)$ from the crack tip (region (c) in fig. 1).

\subsection{Plastic dissipation at the crack tip}
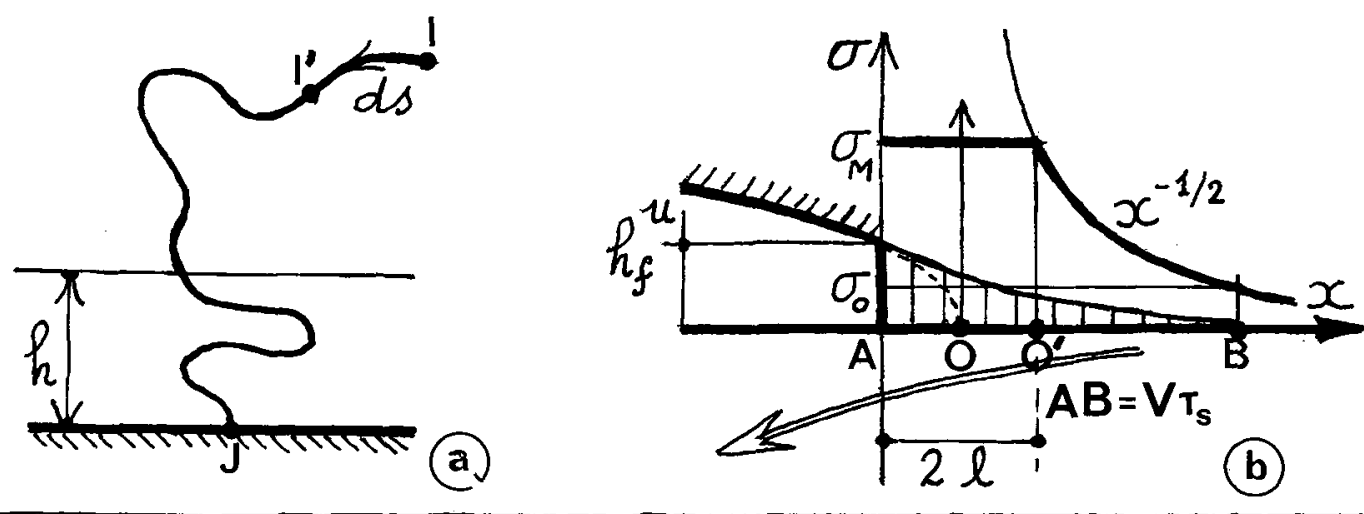

Fug. 2(a): Chain from I to I' and formation of a "primary" layer of height $h$. The primary chain is attached to the substrate at bond $\mathrm{J}$. 2(b) : Displacement $u(x)$ and stresse profile, $\sigma(x)$, at the crack tip.

Chain suction develops from B to A, ahead of the IRWIN crack blunted at A. 
In the chain suction mechanism developped by DE GENNES [7], fracture occurs by suction of the "primary" chain attached to the metallic oxide at J, fig. 2a, which disentangles in reptating out of the polymer bulk, either once the bond I with another chain breaks at a definite stress $\sigma_{0}$, or once simply the free end I starts creeping along the chain tube at a definite yield stress $\sigma_{0}$. This stress value is reached at point $B$ ahead of the crack tip A, fig. $2 b$, i.e. the suction starts at $B$ and ends at $A$, so that, taking again a crack at rest in a medium flowing at rate $\mathrm{V}, \mathrm{AB}=\mathrm{V} \tau_{\mathrm{s}}$, where $\tau_{\mathrm{s}}$ is the time taken by this suction to free completely the chain out of its tube, or "suction time". Finally, the release rate of energy at a moving IRWIN crack is [6] :

$$
\mathrm{G}=\sigma(\mathrm{A}) \cdot \mathrm{h}_{\mathrm{f}}
$$

with $\sigma(A)$ the maximum stress at crack tip $\sigma(A)=\sigma_{M}$ and $h_{f}$ the crack opening displacement (C.O.D.) (fig. 2b). Thus the model introduces three basic parameters : $\sigma(A), h_{f}$ and $\tau_{s}$ we discuss now.

The C.O.D. $h_{f}$ is related to the number of molecular bridges per unit area of junction, $v$, through a volume conservation law : the increase in height dh of the primary layer deposited onto the substrate as a result of chain suction (fig. 2a) stems from the reptated length ds $=$ II' :

$$
\mathrm{dh}=v \mathrm{a}^{2} \mathrm{ds}, \quad \text { or } \quad \mathrm{h}_{\mathrm{f}}=v \mathrm{va}^{2} \mathrm{~L}=v \mathrm{Na}^{3}
$$

with a as a monomer size, $\mathrm{L}$ the chain contour length and $\mathrm{N}$ the number of monomers in the chain, $\mathrm{L}=\mathrm{Na}$. This basic equation assumes no cavitation (crazing) - for crazing one would have simply $h_{f}=\mathrm{Na}$. Now, depending on the case, either $v$ is given by the number of primary chains bound to the metallic oxide and $h_{f} s c a l e s$ as $N$, or $v$ is the number of "cohesive" bulk chains which would have been functionallised; then these chains fulfil the stress free, initial state equation $v \mathrm{Na}^{3}=1 \cdot \mathrm{R}_{0}=\mathrm{a} \sqrt{\mathrm{N}}$ with $\mathrm{R}_{0}$ the coil size, taken here as a "skin" thickness, so that $h_{f}$ scales now as $\sqrt{\mathrm{N}}$.

The stress at crack tip $\sigma_{M}=\sigma(A)$ depends on the crack rate $V$, according to the position of point $B$, fig. $2 b$, with respect to the crack width $\ell_{p}=2 \ell$ (the sharp stress singularity at tip $O$ of a GRIFFITH crack is smeared out in the IRWIN crack into a width $2 \ell$ of constant maximum stress $\sigma_{M}=\mu h_{f} / 2 \ell$, see in fig. 2b). Since $\tau_{s}$ is clearly independent on $V$, the distance $\mathrm{AB}=\mathrm{V} \tau_{\mathrm{s}}$ at which the stress ahead of the tip is $\sigma_{0}$ may exceed the width $\ell_{\mathrm{p}}$, i.e. $\sigma_{\mathrm{M}}$ may exceed $\sigma_{0}$, following the $\sigma \sim x^{-1 / 2}$ dependence of the GRIFFITH stress field. Therefore two cases are to be distinguished:

(i) $V \tau_{s}<l_{p}=\mu h_{f} / \sigma_{0}$, is the quasi static regime where $\sigma(A)=\sigma_{0}$

(ii) $\mathrm{V} \tau_{\mathrm{s}}>\ell_{\mathrm{p}}$ is the fast regime, where $\sigma(\mathrm{A})>\sigma_{0}$. Assuming $\sigma \sim \mathrm{x}^{-1 / 2}$ :

$$
\frac{\sigma(A)^{2}}{\sigma(B)^{2}}=\frac{\sigma(A)^{2}}{\sigma_{0}^{2}}=\frac{V \tau_{s}}{2 l} \text {, with } \sigma(A)=\frac{\mu h_{f}}{2 l}
$$

hence : $\quad \sigma(A)=\left(\sigma_{0}^{2} / \mu\right)\left(V \tau_{s} / h_{f}\right)$

Moreover, the suction mechanism is linear :

$$
\sigma \sim f_{1} \sim(d s / d t) \sim(d h / d t)
$$

since the reptation rate (ds/dt) of a chain under a force $f_{1}$ is linear in $f_{1}$, while $s$ varies as $h$ following equ. (2). Therefore one deduces merely $\sigma=Q(\mathrm{dh} / \mathrm{dt}), \mathrm{Q}$ being a friction constant, $Q=N k T /\left(v a^{4} D_{t}\right)$, with $D_{t}$ the monomer diffusion coefficient along the chain tube [7]. This last equation reads also :

$$
\mathrm{dt}=\mathrm{Qdh} / \sigma, \text { or } \tau_{\mathrm{s}} \cong \mathrm{Q} \mathrm{h}_{\mathrm{f}} / \sigma_{0}
$$


which yields, at about a factor of order unity, the suction time $\tau_{s}$. When plugging equ. (4) into equ. (3), one has lastly :

together with :

$$
\sigma(A)=\frac{\sigma_{0}}{\mu} \cdot V Q=\sigma_{0} \frac{V}{V^{*}} \text { with } V^{*}=\frac{\mu}{Q}
$$

$$
\left(\ell / \ell_{0}\right)=\left(\sigma_{0} / \sigma(A)\right)=\left(V^{*} / V\right) \text {. }
$$

In conclusion, $\mathrm{V}^{*}$ appears as the limiting rate ; order of magnitude evaluations [7] give for $V^{*}$ about $100 \mu \mathrm{m} / \mathrm{s}$ for an elastomer $(\mathrm{N} \cong 30)$, down to about 1 or $0.1 \mu \mathrm{m} / \mathrm{s}$ for a glassy polymer $\left(\mathrm{N} \cong 10^{3}\right)$.

In the quasi-static regime, defined as $V \leq V^{*}, \sigma(A)=\sigma_{0}, \ell_{p}=2 \ell_{0}=\mu h_{f} / \sigma_{0}, G$ is given from equ. (1) and (2) as :

primary chains : $\quad G_{0}=v N\left(\sigma_{0} a^{3}\right)$

or, cohesive bulk chains : $\quad G_{0}=a \sigma_{0} \sqrt{N}$

When interpreting the quantity $\sigma_{0} \mathrm{a}^{3}$ as $U_{b}$, a bond energy, the equation (6a) restitutes the old LAKE and THOMAS argument [8] that when one bond breaks in extracting a taut chain of $\mathrm{N}$ bonds, the needed work is not $U_{b}$ but $N U_{b}$. However here $U_{b}$ would be rather the energy of the bond I, which might be lowered by neighbouring impurities. More important, the equation (6a) emphasizes two behaviours of common observation [9] : $G_{0}$ increases as $v$ (e.g. the percentage of interfacial chemical bonds) and as $N$ (e.g. decreases with crosslinking).

In the fast regime, defined as $\mathrm{V}>\mathrm{V}^{*}, \sigma(\mathrm{A})>\sigma_{0}, \ell_{\mathrm{p}}=2 \ell<2 \ell_{0}, \mathrm{G}$ is given from equ. (1), (2) and (4) as :

$$
\mathrm{G}=\left(\sigma_{0}^{2} / \mu\right) \mathrm{V} \tau_{\mathrm{s}}=\mathrm{G}_{0}\left(\mathrm{~V} / \mathrm{V}^{*}\right)
$$

which shows that $G$ scales as $\tau_{s}$, i.e. as $N^{2}$. More surprising, it is seen that $G$ does not depend anymore on the number of interfacial chemical bonds $v$ since $\tau_{s}$ is clearly independent on it. Therefore as the crack rate is increased the linear dependence obtained at low rates should vanish at high rates. This trend has been effectively observed, not on a metal-polymer but on a glass-polymer system [10], a system merely equivalent here.

\subsection{Viscoelastic losses}

From the above discussion, in part 4.1., and referring to the figure 1, the polymer behaves as an elastic solid in region (b) with a modulus $\mu_{\infty}$ and in region (d) with a modulus $\mu_{0}$. In these regions, as noted by DE GENNES [5], the elastic GRIFFITH field should apply, that is to say $\sigma \sim x^{-1 / 2}$, the strain $\varepsilon \sim x^{-1 / 2}$, the elastic displacement $u \sim x^{1 / 2}$, resulting in the product $\sigma u$ independent on $x$. Now, ou turns out to be the fracture energy $G$ :

$$
\begin{aligned}
& \text { at } x_{1}, \sigma_{1}\left(2 u_{1}\right)=\sigma(A)(2 \delta)=\sigma(A) h_{f}=G_{p} \\
& \text { at } x_{2}, \sigma_{2}\left(2 u_{2}\right)=\sigma_{\infty}(2 u(W))=G
\end{aligned}
$$

noting $G_{p}$ the contribution of plastic dissipation, i.e. of region (a), to $G$ (e.g. $G_{p}=G_{0}$, equ. (6a), at low rate), and $\sigma_{\infty}, u(W)$ respectively the stress and displacement at $x=W$, i.e. at the external surface at the end of region (d). Hence $G$ and $G_{p}$ are proportionnal :

$$
\mathrm{G} / \mathrm{G}_{\mathrm{p}}=\sigma_{2} \mathrm{u}_{2} / \sigma_{1} \mathrm{u}_{1}
$$

In contrast to regions (b) and (d), the behaviour law in region (c) is a viscous one :

$$
\sigma=\eta \frac{d}{d t}\left(\frac{d u}{d x}\right)=\eta v\left(d^{2} u / d x^{2}\right)
$$


so that with again $\sigma \sim x^{-1 / 2}$, one has now $u \sim x^{3 / 2}$, that is to say $\sigma u \sim x$. Hence :

$$
\sigma_{2} u_{2} / \sigma_{1} u_{1}=x_{2} / x_{1}=\ell_{R} / l_{N R}=\mu_{\infty} / \mu_{0}
$$

Finally the fracture energy $G$ is given by [5] :

$$
\mathrm{G}=\mathrm{G}_{\mathrm{p}}\left(\mu_{\infty} / \mu_{0}\right)
$$

Accordingly, viscoelastic losses multiply the plastic dissipation by a factor $\left(\mu_{\infty} / \mu_{0}\right)$, which can be as high as ten in a weakly cross-linked elastomer in the glassy transition. Thus the higher is the modulus drop, the higher is the adherence force.

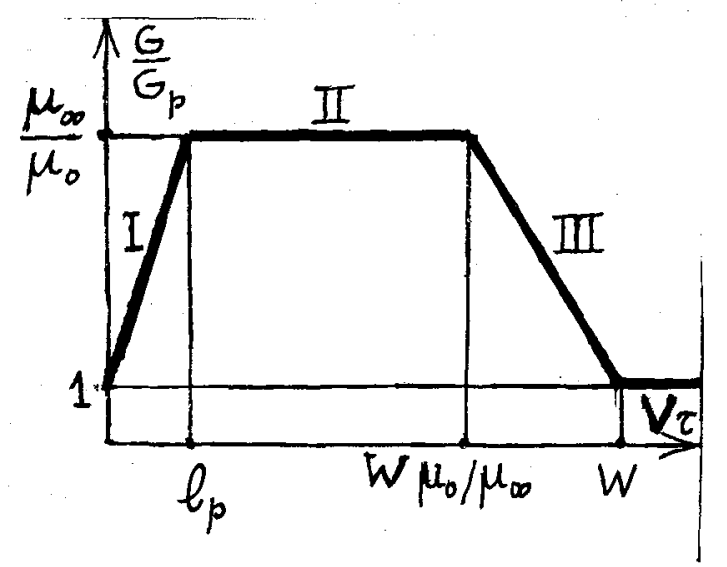

Fig. 3 : The rate dependence of viscoelastic losses [5]
This effect leads to a hump in the $G$ variation with crack rate $V$, as represented in fig. 3 [5]. This is because from the four boundaries appearing in fig. 1 , two are fixed ones : $x=\ell p$, the plastic extension, and $x=W$ the sample width at the end of region (d); and two are V-dependent, $x_{1}=\ell_{\mathrm{NR}}=\mathrm{V} \tau$ and $x_{2}=$ $\ell_{R}=V \tau\left(\mu_{\infty} / \mu_{0}\right)$. Therefore, depending on $\mathrm{V}$, the dissipative region (c) may be partially suppressed as it is seen from the following cases :

- (i) When $l_{N R}=V \tau<l_{p}$, region (b) disappears and region (c) partly merges with (a). The part $I$ in fig. 3 follows.

- (ii) When $\ell_{N R}=V \tau>\ell_{P}$ and when $l_{R}=x_{2}<W$, i.e. $V \tau<W\left(\mu_{0} / \mu_{\infty}\right)$, viscoelastic losses occur in the entire region (c), resulting into a maximum of dissipation, given by equ. (9), and represented by the part II in fig. 3.

- (iii) When $l_{R}=x_{2}>W$, or $V \tau>W\left(\mu_{0} / \mu_{\infty}\right)$, region (d) disappears and region (c) is partly cut off. In equ. (8), $x_{2}$ is to be replaced by $W$, so that $G / G_{p}=W / V \tau$, which shows that $G$ decreases as $V^{-1}$ and becomes equal to $G_{p}$ when $V \tau=W$; it is shown in fig. 3 by the part III. This can be the case for thin films, $W=100 \mu \mathrm{m}$, for laboratory rates : with $\mu_{0} / \mu_{\infty}=0.1, \tau=1 \mathrm{~s}$, one has here $\mathrm{V}>10 \mu \mathrm{m} / \mathrm{s}$.

ROMERO in the work quoted above [3] has observed by peeling experiments parts I and II of the $G(V)$ diagram in fig. 3, with a threshold $V_{c}=\ell_{p} / \tau \cong 10 \mu \mathrm{m} / \mathrm{s}$ and a maximum $G$ value, $\mathrm{G} \cong 10^{3} \mathrm{~J} / \mathrm{m}^{2}$. Assuming current values in the glass transition $\tau=1 \mathrm{~s}$ and $\mu_{\infty} / \mu_{0}=10$ (these quantities have not been measured), would yield $\ell_{p} \cong 10 \mu \mathrm{m}, G \cong 10 G_{0}$ or $G_{0} \cong 100 \mathrm{~J} / \mathrm{m}^{2}$. The following values have been measured : $\sigma_{0} \cong 30 \mathrm{MPa}, \mu \cong 200 \mathrm{MPa}$; so that $2 \delta=$ C.O.D. $=$ $\ell_{\mathrm{p}} \sigma_{0} / \mu \cong 1.5 \mu \mathrm{m}$, which value is consistent with $2 \delta=\mathrm{G}_{0} / \sigma_{0} \cong 3 \mu \mathrm{m}$ obtained from the above estimations. Finally, following equ. (2), $\mathrm{h}_{\mathrm{f}}=2 \delta=\mathrm{Na}\left(\mathrm{va}^{2}\right)$, with $\mathrm{va}^{2}$ equal to a few tenth (grafting is as high as $20 \%$ in wt); this equation taken for $h_{f} \cong 1 \mu \mathrm{m}$ and $a \cong 0.5 \mathrm{~nm}$ would yield $\mathrm{N} \equiv 4$ to $10.10^{3}$, which compares well with the measured (polydisperse) PP molecular weight, NPP $\cong 5000$. Sumarizing, this whole set of values seems to be consistent with a mechanism of cohesive fracture by extraction of the copolymeric PP-PAA chains. These 
chains are effectively the ones found by IR examination on the metallic surface after peeling.

\section{Some final remarks on the mechanical performances of the polymer/metal junction}

We limit here to some final remarks on a few features of polymer/metal junctions observed in practice.

Firstly, the above discussion shows that the cohesive character commonly observed in junction fracture is expected to follow quite directly from the chain suction mechanism ; moreover the strong chemical bonds usually developped at the interface, stronger than the Van der Waals cohesive bonding currently found in polymers, facilitate this situation. At all events, it is seen that even cohesive fracture situations can still be enlightening for adhesion mechanisms.

Secondly, we have not discussed here mechanical interlocking. The corresponding adherence force may be quite strong in practice when experimental conditions are favourable : a large and deep open porosity in the metallic oxide layer (providing it is well adhering to the metal, which may not be the case), a high fluidity of the primary adhesive and a high applied pressure.

Finally, a good adhesive behaviour requires a mechanical homogeneity between the two jointed layers as high as possible, in order to minimize strain gradients under the application of comparable stresses on the two materials, as imposed for example during forming. Considering polymers and metals, and contrary to a popular belief, the observed good formability of metal-polymer sandwiches - sometimes better than the one of the components - is not at all due to an assumed elastomeric state of the polymer related to a hypothetic heating above its $\mathrm{Tg}$. In this situation, the tremendous strain discontinuity though the junction would destroy it. In contrast a possible explanation follows from two arguments :

(i) the high strain rate of forming processes. At a given strain, flow stresses in polymers are much lower than in metals, at least at room temperature and laboratory uniaxial strain rates. However, flow stresses in polymers are strongly thermally activated, while being much less activated in metals (at least for f.c.c. or h.c.p. metals ; b.c.c. metals like Fe do present a high activation also). It follows that the higher is the strain rate, the closer is the flow stress in the polymer from the one in the metal.

(ii) forming tools develop a high hydrostatic pressure in materials ; while again in metals, the flow stress does not change appreciably, it increases in polymers by a factor ten at least at the applied pressure values.

In conclusion, it is quite probable that metal-polymer sandwiches have a good forming potential because flow stresses for comparable strains in these two materials reach comparable values.

\section{REFERENCES}

[1] MAUGIS D., in Microscopic aspects of adhesion and lubrification, J.M. GEORGES Ed., Elsevier Sci. Publ., Amsterdam, (1982) p. 221.

[2] LIENG-HUANG LEE, in Fundamentals of Adhesion, LIENG-HUANG LEE Ed., Plenum Press Publ., New York (1991); [2a] p. $50 ;[2 b]$ p. $11 ;[2 c]$ p. $21,33$.

[3] ROMERO M.A., Doctorat Thesis, University of Lyon I, (1992). Also, M.A. ROMERO, B. CHABERT, and A. DOMARD, J. Appl. Polym. Sci. 47 (1993) 543.

[4] LECAYON G., VIEL P., LE GRESSUS C., BOIZIAU C., LEROY S., and REYNAUD C., J. Chim. Phys. 84 (1987) 259.

[5] DE GENNES P.G., C.R. Acad. Sci. Paris, 307 Série II (1988) 1949. 
[6] FRANCOIS D., PINEAU A., and ZAOUI A., in Comportement Mécanique des Matériaux, Hermès Publ., Paris (1993) p. 350.

[7] DE GENNES P.G., J. Phys. France 50 (1989) 2551 ; also, C.R. Acad. Sci. Paris, 309 Série II 939 and 1125.

[8] LAKE G.J. and THOMAS A.G., Proc. Roy. Soc., A 300 (1967) 108.

[9] CHANG R.J. and GENT A.N., J. Polymer Sci., Polym. Phys. Ed., 19 (1981) 1619.

[10] AHAGON A., and GENT A.N., J. Polymer Sci., Polym. Phys. Ed., 13 (1975) 1285. 